\title{
An Energy Efficient Cognitive Radio System with Quantized Soft Sensing and Duration Analysis
}

\author{
AbdulRahman Alabbasi and Basem Shihada \\ Computer, Electrical and Mathematical Science and Engineering (CEMSE) Division \\ King Abdullah University of Science and Technology (KAUST) \\ Email: \{abdulrahman.alabbasi, basem.shihada\}@kaust.edu.sa
}

\begin{abstract}
In this paper, an energy efficient cognitive radio system is proposed. The proposed design optimizes the secondary user transmission power and the sensing duration combined with soft-sensing information to minimize the energy per goodbit. Due to the non-convex nature of the problem we prove its pseudo-convexity to guarantee the optimal solution. Furthermore, a quantization scheme, that discretize the softsensing information, is proposed and analyzed to reduce the overload of the continuously adapted power. Numerical results show that our proposed system outperforms the benchmark systems. The impact of the quantization levels and other system parameters is evaluated in the numerical results.
\end{abstract}

Index Terms-Spectrum sharing, Energy efficiency, Spectrum sensing, Resource allocation,

\section{INTRODUCTION}

Carbon dioxide footprint effect derives the communication systems to consider energy efficiency into their design [1]. Cognitive radio (CR) technology is among these systems, which is proposed to overcome the inefficient use of frequency spectrum and its resulting scarcity [2]. The combination of cognitive systems and energy efficiency technologies can be implemented in several ways as presented in [3]-[6].

Researchers have been considering optimal energy efficient systems by minimizing the system energy while preserving certain quality of service (QoS) parameters, such as rate, delay, etc. However, it has been shown in [7] that optimizing the Energy Per Goodbit (EPG) yields to an improvement results in terms of energy efficiency. Furthermore, authors in [8] proposed a cooperative sensing optimized fusion rule threshold based on k-out-of-N rule that maximizes the energy efficiency (e.g., throughput over energy metric). This work shows that the optimized k-out-of-N based rule outperforms both the AND and OR rules in terms of energy efficiency. Another work, in [9], has investigated this rule but using the golden section search method to maximize energy efficiency. In [10], the impact of sensing on EPG is tackled, without considering the sensing duration under a single-input single-output (SISO) orthogonal frequency-division mutiplexing (OFDM) system. A joint design of the optimal sensing duration and transmission duration is proposed in [11]. Where the authors considered a throughput per energy as their energy efficiency metric. The primary user (PU) protection is guaranteed by limiting the detection probability within a certain threshold. The effect of the sensing errorness and correctness probabilities on CR multiple-input multiple-output (MIMO) systems is considered in [12] and [6].

In this work, we propose and analyze an energy efficient CR system with spectrum sharing utilizing soft-sensing information (SSI) about PU. The proposed scheme optimizes both the secondary user (SU) transmission power and sensing time, consequently, the communication time. In our analysis, we consider PU to freely switch between active and idle status based on Bernoulli distribution, with probability $\alpha_{1}$. An on-board sensor is assumed to be available at the SU side. The secondary transmitter (ST) utilizes the collected SSI, from SU's sensor, to adaptively access the PU channel within the tolerable interference threshold. Additionally, it optimizes the corresponding sensing duration. Furthermore, a quantization scheme for the SSI is proposed and analyzed.

Our work has several unique contributions as follows. We consider EPG metric with SSI to evaluate the system performance, where the authors in [11] considered throughput per energy metric without SSI. Unlike [8], [9], [11], we proved the pseudo-convexity of the proposed formulation. This leads to the fact that satisfying the associated Karush-Kuhn-Tucker (KKT) conditions guarantees the global optimality of the solution. Optimal analytical results are provided for the SU transmission power. Furthermore, it is shown that the verified problem structure allows us to utilize either the alternating directing method (ADM) algorithm or back-fitting algorithm to obtain the global optimal solution. In our formulation, a SU minimum average rate constraint and primary receiver $(\mathrm{PR})$ average interference protection constraint are enforced to the problem. Furthermore, the detection and false alarm probabilities are bounded by a certain threshold. However, both [8] and [9] did not consider either the minimum average throughput constraint or the PR average interference protection constraint. The numerical results shows the improvement of the proposed SSI based scheme compared to the one without SSI. Furthermore, it shows the impact of increasing the number of quantization levels on the system EPG performance.

This paper is organized as follows. Section II describes our system model with some related background on PU sensing approach. Section III discusses the problem formulation while analyzing its structure. Section IV considers the optimal solution. Section V discusses the proposed SSI quantization scheme. Finally, selected numerical results are presented in Section VI.

\section{System Model ANd RELATEd BACKGRound}

\section{A. System Model}

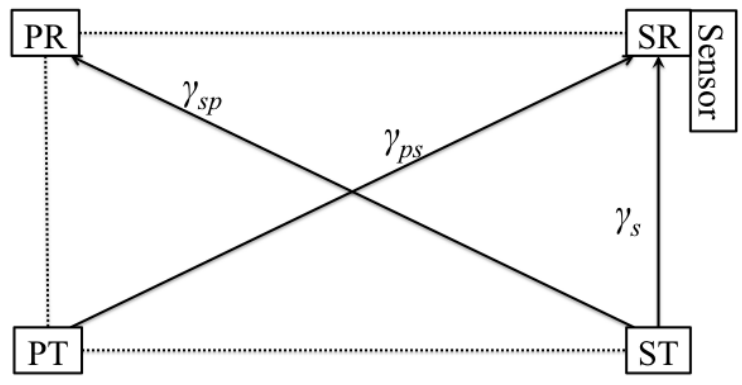

Fig. 1. System Model.

The proposed system consists of a pair of SUs and a pair of PUs as shown in Figure 1. Primary transmitter (PT) to secondary receiver (SR) channel, ST to PR channel, and ST to SR channel are designated as $\gamma_{p s}, \gamma_{s p}$, and $\gamma_{s}$. General channel distribution is assumed in the analysis, unless otherwise is mentioned. The fading 


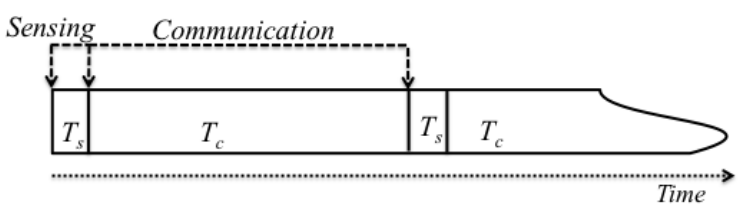

Fig. 2. Sensing and Communication Intervals.

channels are assumed to be independent. Note that we assume the availability of a real-time spectrum sensor at the SR. The sensor produces sensing information which helps in the resource allocation, (i.e., power and sensing time).

Our design optimizes the system's resources (i.e., power, communication and sensing times), to achieve the maximum energy efficiency (minimum EPG). In our system, the sensor senses the channels for $T_{s}$ then start transmitting data for a period of time $T_{c}$, such that, $T=T_{c}+T_{s}$, as shown in Figure 2. We don't enforce any limitation of the sensing time except the ones that affect the communication rate and quality of sensing. Additionally, we assume that the channel coherence time is large enough such that it does not change within two sensing periods. Perfect channel state information (CSI) knowledge on the secondary channel, $\gamma_{s}$, is assumed, whereas, average CSI knowledge about $\gamma_{s p}$ is considered, (i.e., $\bar{\gamma}_{s p}$ ). The secondary channel, $\gamma_{s}$, and the SSI, $\eta\left(\zeta, T_{s}\right)$, to be described later, are shared between ST and SR through a limited delay, error free feedback channel. The interference form PT to SR is considered as noise, (i.e., we do not implement any interference cancelation scheme such as, the successive interference cancelation). It is assumed that the interference from ST toward PR is limited to a threshold, such that the PU's tolerance level is not violated. Note that we assume an Additive White Gaussian Noise (AWGN) noise at SR. SU's transmission power and sensing time are considered as optimization variables that affect the energy efficiency performance of the system, whereas, PU's transmission power is fixed.

\section{B. Sensing Background}

This section provides a background on the sensing information. We show the effect of sensing information on the system performance which appears in different points, as follows. First, the effect of the sensing duration and the corresponding power required by the sensing process. Second, the accuracy of sensing error and its effect on the rate. Finally, the effect of sensing accuracy on the secondary to primary interference constraint. Note that all the expectation of the energy efficiency metric and the enforced constraints are over both the secondary channel and the sensing information.

In this system, we consider an energy detector based sensing, since we do not have a prior knowledge about PU signal or channel. As known in the literature, the output of the energy detector is derived as follows, [13],

$$
\zeta\left(T_{s}\right)=\frac{1}{T_{s}} \sum_{n=1}^{T_{s}}\left|y_{i}(n)\right|^{2},
$$

where $T_{s}$ is the number of sensing samples. The received signal at SR sensor, $y_{i}(n)$, is defined as,

$$
y_{i}(n)=\left\{\begin{array}{lll}
w_{i}(n) & \mathcal{H}_{0}: & (\mathrm{PU} \text { is idle) } \\
h_{p s i}(n) x_{i}(n)+w_{i}(n) & \mathcal{H}_{1}: & \text { (PU is active) }
\end{array},\right.
$$

where, $x_{i}(n)$ is the PU data, and $w_{i}(n)$ is the AWGN, with zero mean and unity variance, at the sensor side.

Authors of [13] have done a thorough investigation on the Probability Density Function (PDF) of $\zeta\left(T_{s}\right)$ for both cases $\mathcal{H}_{0}$ and $\mathcal{H}_{1}$. Following the same general approach, we obtain the PDF of both $\mathcal{H}_{0}$ and $\mathcal{H}_{1}$ as $g_{0}(\zeta)$ and $g_{1}(\zeta)$. Deciding whether PU is active or idle requires an optimal decision mechanism, as follows,

$$
\begin{aligned}
& \text { If: } \zeta>\gamma_{t h}, \text { Decide } \mathcal{H}_{1} \\
& \text { If: } \zeta \leq \gamma_{t h} \text {, Decide } \mathcal{H}_{0} \text { ' }
\end{aligned}
$$

where $\gamma_{t h}$ is the threshold which identifies the existence of the PU. It follows that the correctness and errorness probabilities are expressed as follows,

$$
P_{F A}^{c}\left(T_{s}\right)=\int_{-\infty}^{\gamma_{t h}} g_{0}(\zeta) d \zeta, P_{M D}\left(T_{s}\right)=\int_{-\infty}^{\gamma_{t h}} g_{1}(\zeta) d \zeta .
$$

Another formulation of the errorness and correctness probabilities is considered as the exponential distribution of the log-likelihood function of both hypotheses PDFs [14].

In addition to the effect of the errorness and correctness probabilities, the SSI has a major impact on the system performances. The SSI metric is formulated as the likelihood function of the PDFs, (i.e., $\mathcal{H}_{1}$ and $\mathcal{H}_{0}$ ), as follows,

$$
\eta\left(\zeta, T_{s}\right)=\frac{g_{0}(\zeta)}{g_{1}(\zeta)}
$$

This SSI is utilized in the adaptation of the transmission power and the sensing and communication durations. Other work in [8], [9], [11], have considered cooperative sensing under the energy efficiency theme. The impact of this consideration reflects on (4) and (5). Therefore, without loss of generality, we consider the sensing information resulted from one sensor, knowing that this results can be easily expanded to the cooperative sensing case.

\section{PRoblem Formulation}

In this section, the problem formulation of the energy efficiency system and related analysis of the extended problem are described. The proposed system minimizes the EPG metric, subject to several constraints, namely, minimum average rate constraint, average interference constraint, peak power constraint, and a sensing duration lower bounds constraint.

$$
\begin{array}{rl}
\mathbb{P}: \min _{P_{s}(\gamma), T_{s}} & E=\frac{\mathbb{E}_{\gamma, \zeta}\left\{T_{c} P_{t} P_{s}(\gamma)+P_{c}+T_{s} P_{s n s}\right\}}{\mathbb{E}_{\gamma, \zeta}\left\{T_{c} \log \left(1+\frac{P_{s}(\gamma) \gamma_{s}}{1+P_{p} \gamma_{p s}}\right)\right\}} \\
\text { s.t. } \quad \mathfrak{C}_{1}: \mathbb{E}_{\gamma, \zeta}\left\{T_{c} \log \left(1+\frac{P_{s}(\gamma) \gamma_{s}}{1+P_{p} \gamma_{p s}}\right)\right\} \geq R_{m i n} \\
\\
\mathfrak{C}_{2}: \mathbb{E}_{\gamma, \zeta \mid \mathcal{H}_{1}}\left\{P_{s}(\gamma) \gamma_{s p}\right\} \leq Q_{\text {int }} \\
\\
\mathfrak{C}_{3}: P_{s}(\gamma) \leq P p k \\
\mathfrak{C}_{4}: T_{s} \geq \max \left[\left[\frac{Q^{-1}\left(P_{F A}^{c}\right)}{f_{0}}\right]^{2},\left[\frac{Q^{-1}\left(P_{M D}\right)}{f_{1}}\right]^{2}\right]
\end{array}
$$

where, $P_{s}(\gamma)$ and $P_{p}$ are the transmission powers of SU and PU, respectively. The parameters $Q_{i n t}, P_{p k}$, and $R_{m i n}$ are assigned constants for the maximum limit of the average interference at PR from ST, the maximum peak power of SU, and the minimum acceptable SU average rate. The constants $P_{c}$ and $P_{t}$ are assigned to the circuit power of the radio devices and power amplifier constant power consumption. $P_{F A}^{c}$ and $P_{M D}$ are constants refer to the desired false alarm complement and miss detection. The variable $\gamma$ refers to both the SU channel, $\gamma_{s}$, and ST to PR channel, $\gamma_{s p}$. Whereas, the variable $\zeta$ refers to the sensing information produced from the sensor.

In $\mathbb{P}$, the objective function describes the average EPG which includes the effect of adaptive sensing duration, sensing accuracy, 
and adaptive power allocation. The problem constraints are described as follows. The minimum average SU rate constraint, (6b), takes into account the interference from PT as noise, and keep the SU average rate above a constant threshold. Constraint (6c) limits the average ST to PR interference to a certain tolerable threshold. Constraint (6d) presents the peak power constraint which prevents the power amplifier from operating under the saturation region. Finally, constraint (6e) bounds the sensing duration to achieve a certain miss-detection and false alarm probabilities.

The impact of sensing information on problem $\mathbb{P}$ is hidden in the expectations. This effect is shown by expanding the expectations with respect to the averaging variable $\zeta$ and both PU existence hypotheses, $\mathcal{H}_{1}$ and $\mathcal{H}_{0}$. Problem $\mathbb{P}$, after expansion, is equivalent to the following,

$$
\begin{aligned}
\mathbb{P}_{0}: \min _{P_{s}(\gamma), T_{s}} & E_{0} \\
\text { s.t. } & \mathfrak{C}_{1}: R_{0} \geq R_{\text {min }} \\
& \mathfrak{C}_{2}: \alpha P_{M D}\left(T_{s}\right) \mathbb{E}_{\gamma, \zeta \mid \mathcal{H}_{1}}\left\{P_{s}(\gamma) \gamma_{s p}\right\} \leq Q_{\text {int }} \\
& \mathfrak{C}_{3} ; \mathfrak{C}_{4},
\end{aligned}
$$

where, $\alpha_{0}=1-\alpha, E_{0}$ is defined in (9), and $R_{0}$ is defined in (10). It is worth noting that both $E_{0}$ and $R_{0}$ show the impact of the sensing accuracy and probability of both hypotheses $\mathcal{H}_{0}$ and $\mathcal{H}_{1}$. Later on, when solving the problem, the impact of the SSI parameter $\eta\left(\zeta, T_{s}\right)$ on both $E_{0}$ and $R_{0}$ is introduced. The power policy that is considered to change problem $\mathbb{P}$ to $\mathbb{P}_{0}$ is expressed as follows,

$$
P_{s}(\gamma)^{*}=\left\{\begin{array}{ll}
0, & \zeta>\gamma_{t h} \\
P_{s}(\gamma) & \zeta \leq \gamma_{t h}
\end{array} .\right.
$$

This policy explains the reason of having only two probabilities, $P_{M D}\left(T_{s}\right)$ and $P_{F A}^{c}\left(T_{s}\right)$, in both $R_{0}$ and $E_{0}$ terms.

Problem $\mathbb{P}_{0}$ is a fractional non-convex function with respect to both optimization variables $P_{s}(\gamma)$ and $T_{s}$. In order to solve $\mathbb{P}_{0}$, we begin by investigating the problem structure with respect to each of the optimization variables. Finding an optimal solution to $\mathbb{P}_{0}$ with respect to each variable is beneficial in proposing an iterative algorithm that obtain a global optima solution [17]. It is known that by satisfying the KKT conditions a global optimal solution is obtained for a convex problem (i.e., convex objective function with convex constraint). A generalized version of this result is stated in the following theorem [15][Sec. 4.4]. Prior to the theorem it is necessary to state the following definition.

Definition Problem $\mathcal{P}$ is defined as follows,

$$
\mathcal{P}: \min _{x} f(x), x \in S=\left\{x \in X: g_{i}(x) \leq 0, i=1, \ldots, m\right\} .
$$

This definition can be easily applied to the case of problem $\mathbb{P}_{0}$.

Theorem III.1. Consider problem $\mathcal{P}$ with $x^{*}$ as a feasible point. Let $f$ be pseudo-convex at $x^{*} \in S$ and that $g_{i}$ are differentiable and quasi-convex at $x^{*}$. If there exist $\lambda_{i} \in \mathbb{R}$, such that, the KKT conditions are satisfied,

$$
\left\{\begin{array}{l}
\nabla f\left(x^{*}\right)+\sum_{i=1}^{m} \lambda_{i} \nabla g_{i}\left(x^{*}\right)=0 \\
\lambda_{i} \geq 0, i=1, \ldots, m \\
\lambda_{i} g_{i}\left(x^{*}\right)=0, i=1, \ldots, m
\end{array},\right.
$$

then $x^{*}$ is a global minimum point for $\mathcal{P}$.

Proof: The proof is stated in [15][Sec. 4.4].

Theorem III.1 is utilized to solve problem $\mathbb{P}_{0}$. The following lemma is deducted to show that problem $\mathbb{P}_{0}$, (7), satisfies the settings stated in Theorem III.1. For notation simplicity, let $P_{s}(\gamma)=P_{s}$. lemma III.2. Let $P_{s}^{*}$ be a feasible solution, of problem $\mathbb{P}_{0}$, that satisfies the KKT conditions. It follows that $P_{s}^{*}$ is a global optimal solution for problem $\mathbb{P}_{0}$.

Proof: To proof this lemma, it is essential to verify that the objective function of problem $\mathbb{P}_{0}$ is pseudo-convex function with respect to $P_{s}$. The corresponding constraints must be verified to have quasi-convex structure with respect to $P_{s}$. It is clear that all the constraint are convex ones with respect to $P_{s}$. Consequently, they are quasi-convex constraints. To show that $E_{0}\left(P_{s}\right)$ is pseudo-convex, two conditions must be satisfied [15]. Note that, hereafter, $E_{0}\left(P_{s}\right)$ and $E_{0}$ are used alternately depending on the context.

1) $E_{0}\left(P_{s}\right)$ is a quasi-convex function on its domain $\mathcal{S}$.

2) If $P_{s}^{*} \in \mathcal{S}, \nabla E_{0}\left(P_{s}^{*}\right)=0$, then $P_{s}^{*}$ is a local minimum for $E_{0}$.

The function $f(x)$ is quasi-convex iff $f\left(\lambda x^{(1)}+(1-\lambda) x^{(2)}\right) \leq$ $\max \left(f\left(x^{(1)}\right), f\left(x^{(2)}\right)\right)$. To show the quasi-convexity structure of $E_{0}\left(P_{s}\right)$, we write it as, $E_{0}\left(P_{s}\right)=\frac{E_{n}\left(P_{s}\right)}{E_{d}\left(P_{s}\right)}$. Convexity and concavity properties of each $E_{n}$ and $E_{d}$, with respect to $P_{s}$, are utilized to show the quasi-convexity of $E_{0}$, as follows (for notation simplicity, let $P_{s}(\gamma)=P_{s}$ and $\left.E_{0}\left(P_{s}^{(2)}\right) \leq E_{0}\left(P_{s}^{(1)}\right)\right)$,

$$
\begin{aligned}
& E_{n}\left(\lambda P_{s}^{(1)}+(1-\lambda) P_{s}^{(2)}\right)=\lambda E_{n}\left(P_{s}^{(1)}\right)+(1-\lambda) E_{n}\left(P_{s}^{(2)}\right) \\
& \stackrel{(a)}{\leq} \lambda E_{n}\left(P_{s}^{(1)}\right)+(1-\lambda) \frac{E_{n}\left(P_{s}^{(1)}\right)}{E_{d}\left(P_{s}^{(1)}\right)} E_{d}\left(P_{s}^{(2)}\right) \\
& \stackrel{(b)}{\leq} \frac{E_{n}\left(P_{s}^{(1)}\right)}{E_{d}\left(P_{s}^{(1)}\right)}\left[E_{d}\left(\lambda P_{s}^{(1)}+(1-\lambda) P_{s}^{(2)}\right)\right] \\
& \Longrightarrow E_{0}\left(\lambda P_{s}^{(1)}+(1-\lambda) P_{s}^{(2)}\right) \leq E_{0}\left(P_{s}^{(1)}\right),
\end{aligned}
$$

where (a) results from assuming that $E_{0}\left(P_{s}^{(2)}\right) \leq E_{0}\left(P_{s}^{(1)}\right)$, (b) is valid because of the concave property of $E_{d}$. Based on the assumption $E_{0}\left(P_{s}^{(2)}\right) \leq E_{0}\left(P_{s}^{(1)}\right)$, the result shows that $E_{0}\left(P_{s}\right)$ is a quasiconvex function.

The second step in proving the pseudo-convexity of $E_{0}\left(P_{s}\right)$, is to show that $\nabla E_{0}\left(P_{s}^{*}\right)=0$. It is clear that $\nabla E_{0}\left(P_{s}^{*}\right)=0$ iff $\nabla E_{n}\left(P_{s}^{*}\right) E_{d}\left(P_{s}^{*}\right)-E_{n}\left(P_{s}^{*}\right) \nabla E_{d}\left(P_{s}^{*}\right)=0$. Then, $\nabla E_{n}\left(P_{s}^{*}\right)=$ $E_{0}\left(P_{s}^{*}\right) \nabla E_{d}\left(P_{s}^{*}\right)$. Utilizing the properties of $E_{n}$ function,

$$
\begin{aligned}
E_{n}\left(P_{s}\right) & \stackrel{(a)}{\geq} E_{n}\left(P_{s}^{*}\right)+\nabla E_{n}\left(P_{s}^{*}\right)\left(P_{s}-P_{s}^{*}\right) \\
& \geq E_{n}\left(P_{s}^{*}\right)+E_{0}\left(P_{s}^{*}\right) E_{d}\left(P_{s}\right)-E_{n}\left(P_{s}^{*}\right) \\
& \Longrightarrow \frac{E_{n}\left(P_{s}\right)}{E_{d}\left(P_{s}\right)} \geq E_{0}\left(P_{s}^{*}\right),
\end{aligned}
$$

where (a) follows from the convex property of $E_{n}$. This result shows that any stationary point of function $E_{0}$ is a global minimum.

By combining (13) and (14), it is clear that the function $E_{0}\left(P_{s}\right)$ is pseudo-convex. Utilizing Theorem III.1, the global optimal solution of problem $\mathbb{P}_{0}$, with respect to $P_{s}(\gamma)$, is obtained by satisfying the KKT conditions of problem $\mathbb{P}_{0}$.

After obtaining a specific structure of problem $\mathbb{P}_{0}$ with respect to $P_{s}(\gamma)$ through which an optimal $P_{s}$ can be derived a similar technique should be applied to find the optimal $T_{s}$. Since it is difficult to show an explicit formula for the optimal $T_{s}$, we utilize the well known bisection algorithm. In order to utilize the bisection algorithm it is a must to show the quasi-convexity structure of $E_{0}$ with respect to $T_{s}$ [16]. This structure is proved by deriving the determinant of the Bordered Hessian matrix, and verify that it is negative. Another way is to show that $E_{0}$ is decreasing at $T_{s} \rightarrow 0$, then, it is increasing at $T_{s} \rightarrow \infty$. The numerator of the derivative of $E_{0}$ is derived in (15), 


$$
\begin{gathered}
E_{0}=\frac{E_{n}}{E_{d}}=\frac{T_{c} P_{t}\left[\alpha_{0} P_{F A}^{c}\left(T_{s}\right) \mathbb{E}_{\gamma, \zeta \mid \mathcal{H}_{0}}\left\{P_{s}(\gamma)\right\}+\alpha P_{M D}\left(T_{s}\right) \mathbb{E}_{\gamma, \zeta \mid \mathcal{H}_{1}}\left\{P_{s}(\gamma)\right\}\right]+P_{c}+T_{s} P_{s n s}}{T_{c}\left[\alpha_{0} P_{F A}^{c}\left(T_{s}\right) \mathbb{E}_{\gamma, \zeta \mid \mathcal{H}_{0}}\left\{\log \left(1+P_{s}(\gamma) \gamma_{s}\right)\right\}+\alpha P_{M D}\left(T_{s}\right) \mathbb{E}_{\gamma, \zeta \mid \mathcal{H}_{1}}\left\{\log \left(1+\frac{P_{s}(\gamma) \gamma_{s}}{1+P_{p} \gamma_{p s}}\right)\right\}\right]} \\
R_{0}=T_{c}\left[\alpha_{0} P_{F A}^{c}\left(T_{s}\right) \mathbb{E}_{\gamma, \zeta \mid \mathcal{H}_{0}}\left\{\log \left(1+P_{s}(\gamma) \gamma_{s}\right)\right\}+\alpha P_{M D}\left(T_{s}\right) \mathbb{E}_{\gamma, \zeta \mid \mathcal{H}_{1}}\left\{\log \left(1+\frac{P_{s}(\gamma) \gamma_{s}}{1+P_{p} \gamma_{p s}}\right)\right\}\right]
\end{gathered}
$$

$$
\begin{aligned}
\frac{\partial E_{0}}{\partial T_{s}}= & {\left[P_{s n s}+T_{c} P_{t}\left(\tilde{P}_{s 0} \alpha_{0} P_{F A}^{\prime c}\left(T_{s}\right)+\tilde{P}_{s 1} \alpha P_{M D}^{\prime}\left(T_{s}\right)\right)-P_{t}\left(\tilde{P}_{s 0} \alpha_{0} P_{F A}^{c}\left(T_{s}\right)+\tilde{P}_{s 1} \alpha P_{M D}\left(T_{s}\right)\right)\right] } \\
& {\left[T_{c}\left(C_{o f f} \alpha_{0} P_{F A}^{c}\left(T_{s}\right)+C_{o n} \alpha P_{M D}\left(T_{s}\right)\right)\right] } \\
& -\left[-\left(C_{o f f} \alpha_{0} P_{F A}^{c}\left(T_{s}\right)+C_{o n} \alpha P_{M D}\left(T_{s}\right)\right)+T_{c}\left(C_{o f f} \alpha_{0} P_{F A}^{\prime c}\left(T_{s}\right)+C_{o n} \alpha P_{M D}^{\prime}\left(T_{s}\right)\right)\right] \\
& {\left[P_{s n s} T_{s}+P_{c}+T_{c} P_{t}\left(\tilde{P}_{s 0} \alpha_{0} P_{F A}^{c}\left(T_{s}\right)+\tilde{P}_{s 1} \alpha P_{M D}\left(T_{s}\right)\right)\right] . }
\end{aligned}
$$

where,

$$
\begin{aligned}
& P_{F A}^{\prime c}\left(T_{s}\right)=\frac{f_{0} e^{\left(\frac{-T_{s} f_{0}^{2}}{2}\right)}}{\sqrt{\left(2 \pi T_{s}\right)}}, P_{M D}^{\prime}\left(T_{s}\right)=\frac{f_{1} e^{\left(\frac{-T_{s} f_{1}^{2}}{2}\right)}}{\sqrt{\left(2 \pi T_{s}\right)}}, \\
& C_{o f f}=\mathbb{E}_{\gamma, \zeta \mid \mathcal{H}_{0}}\left[\log \left(1+P_{s}(\gamma) \gamma_{s}\right)\right], \\
& C_{o n}=\mathbb{E}_{\gamma, \zeta \mid \mathcal{H}_{1}}\left[\log \left(1+\frac{P_{s}(\gamma) \gamma_{s}}{1+P_{p} \gamma_{p s}}\right)\right], \\
& \tilde{P}_{s 0}=\mathbb{E}_{\gamma, \zeta \mid \mathcal{H}_{0}}\left[P_{s}(\gamma)\right], \tilde{P}_{s 1}=\mathbb{E}_{\gamma, \zeta \mid \mathcal{H}_{1}}\left[P_{s}(\gamma)\right] .
\end{aligned}
$$

Applying some calculus manipulation techniques, we obtain,

$$
\lim _{T_{s} \rightarrow 0} \frac{\partial E_{0}}{\partial T_{s}}=-T\left[C_{o f f} \alpha_{0} \frac{f_{0}}{\sqrt{\left(2 \pi T_{s}\right)}}+C_{o n} \alpha \frac{f_{1}}{\sqrt{\left(2 \pi T_{s}\right)}}\right]<0,
$$

which verifies that $E_{0}$ is decreasing at $T_{s} \rightarrow 0$. On the other hand, at $T_{s} \rightarrow \infty$, it follows that,

$$
\begin{aligned}
& \lim _{T_{s} \rightarrow T \rightarrow \infty} \frac{\partial E_{0}}{\partial T_{s}}= \\
& \left(C_{o f f} \alpha_{0} P_{F A}^{c}\left(T_{s}\right)+C_{o n} \alpha P_{M D}\left(T_{s}\right)\right)\left[P_{s n s} T+P_{c}\right]>0 .
\end{aligned}
$$

This verifies that $E_{0}$ is increasing at $T_{s} \rightarrow T \rightarrow \infty$. In order to verify the quasi-convexity structure of $E_{0}$, utilizing (17) and (18), it is necessary to show that $E_{0}$ has a single stationary point. This proof is omitted due to the page limitation. Thus, $E_{0}$ is quasi-convex with respect to $T_{s}$ and by applying a bisection algorithm, we obtain the global optimal $T_{s}$.

\section{Problem Solution}

In this section, the optimal expression of the optimization variable, $P_{s}$, is obtained. To show the analytical solution of the $\mathbb{P}_{0}$ we transform it to the epigraph form. It is shown in [15] that minimizing a convex or pseudo-convex problem is equivalent to minimizing its epigraphic form. The new form of problem $\mathbb{P}_{0}$ is expressed as follows,

$$
\begin{aligned}
& \mathbb{P}_{1}: \quad \min _{P_{s}(\gamma), T_{s}, t} t \\
& \text { s.t. } \quad \mathfrak{C}_{0}: E_{n}-t E_{d} \leq 0 \\
& \mathfrak{C}_{1} ; \mathfrak{C}_{2} ; \mathfrak{C}_{3} ; \mathfrak{C}_{4},
\end{aligned}
$$

where $t$ is the new optimization variable.

The Lagrangian function of problem $\mathbb{P}_{1}$ is constructed while including $\mathfrak{C}_{0}, \mathfrak{C}_{1}$, and $\mathfrak{C}_{2}$. Whereas, the peak power constraint $\mathfrak{C}_{3}$ and the peak sensing time constraint $\mathfrak{C}_{4}$ are left to a later step. The explicit formula of the Lagrangian function is expressed in (20). The Lagrangian multipliers, $\lambda_{0}, \lambda_{1}$, and $\lambda_{2}$ are associated with constraints $\mathfrak{C}_{0}, \mathfrak{C}_{1}$, and $\mathfrak{C}_{2}$, respectively. Note that the corresponding KKT conditions, of constraints $\mathfrak{C}_{0}, \mathfrak{C}_{1}$, and $\mathfrak{C}_{2}$, are expressed in (21), (22), and (23).

$$
\lambda_{2}\left[\mathbb{E}_{\gamma, \zeta \mid \mathcal{H}_{1}}\left\{\alpha P_{M D}\left(T_{s}\right) P_{s}^{*} \gamma_{s p}\right\}-Q_{\text {int }}\right]=0 .
$$

The analytical expression of the optimal SU transmission power, $P_{s}^{*}$, is found by satisfying the KKT conditions, as follows,

$$
P_{s}^{*}=\left[\frac{-b+\sqrt{\left(b^{2}-4 a c\right)}}{2 a}\right]_{0}^{P_{p k}} .
$$

where, $a=\gamma_{s}^{2} \Phi ; b=-\gamma_{s}^{2}\left[\alpha_{0} P_{F A}^{c}\left(T_{s}\right) \eta\left(\zeta, T_{s}\right)+\alpha P_{M D}\left(T_{s}\right)\right]+$ $\Phi \gamma_{s}\left(2+P_{p} \gamma_{p s}\right) ; c=-\gamma_{s} \alpha_{0} P_{F A}^{c}\left(T_{s}\right) \eta\left(\zeta, T_{s}\right)\left(1+P_{p} \gamma_{p s}\right)-$ $\gamma_{s} \alpha P_{M D}\left(T_{s}\right)+\Phi\left(1+P_{p} \gamma_{p s}\right)$. The operator $[z]_{y}^{x}=\max (y, \min (z, x))$. The parameter $\Phi=$ $\frac{\gamma_{u}\left(\alpha, \zeta, T_{s}\right) \lambda_{0} P_{t} T_{c}+\lambda_{2} \bar{\gamma}_{s} \alpha P_{M D}\left(T_{s}\right)}{T_{c}\left(\lambda_{1}+\lambda_{0} t\right)}$ where, $\gamma_{u}\left(\alpha, \zeta, T_{s}\right)=$ $\left[\alpha_{0} P_{F A}^{c}\left(T_{s}\right) \eta\left(\zeta, T_{s}\right)+\alpha P_{M D}\left(T_{s}\right)\right]$. The parameter $\gamma_{u}\left(\alpha, \zeta, T_{s}\right)$ contains the effect of the SSI, $\eta\left(\zeta, T_{s}\right)$. Note that $\gamma_{u}\left(\alpha, \zeta, T_{s}\right)$ is directly proportional to the value of $\Phi$. Depending on other parameters, increasing $\eta\left(\zeta, T_{s}\right)$, thus, increasing $\Phi$, results in changing $P_{s}^{*}$.

Note that if $\alpha P_{M D}\left(T_{s}\right) \mathbb{E}_{\gamma, \zeta \mid \mathcal{H}_{1}}\left\{P_{s}(\gamma) \gamma_{s p}\right\}<Q_{\text {int }}$ is strictly satisfied, $\Phi=\frac{\gamma_{u}\left(\alpha, \zeta, T_{s}\right) \lambda_{0} P_{t} T_{c}}{T_{c}\left(\lambda_{1}+\lambda_{0} t\right)}$. Furthermore, considering the case where the primary transmission power is very low, or the primary to secondary channel is in deep fading, such that $\mathbb{E}_{\gamma, \zeta \mid \mathcal{H}_{0}}\{R\} \approx$ $\mathbb{E}_{\gamma, \zeta \mid \mathcal{H}_{1}}\{R\}$. It follows that $P_{s}(\gamma)$ has water-filling formula as follows,

$$
P_{s}^{*}=\left[\frac{T_{c} \gamma_{u}\left(\alpha, \zeta, T_{s}\right)\left(\lambda_{1}+\lambda_{0} t\right)}{\lambda_{2} \bar{\gamma}_{s p} \alpha P_{M D}\left(T_{s}\right)+\lambda_{0} P_{t} T_{c} \gamma_{u}\left(\alpha, \zeta, T_{s}\right)}-\frac{1}{\gamma_{s}}\right]_{0}^{P_{p k}} .
$$

Note that the value of $\lambda_{0}, \lambda_{1}$, and $\lambda_{2}$ are obtained by numerically solving (21), (22), and (23), respectively.

To solve problem $\mathbb{P}_{0}$ with respect to both variables $P_{s}(\gamma)$ and $T_{s}$, after verifying its structure in Section III, an iterative algorithm must be utilized, such as, ADM algorithm or back-fitting algorithm. The ADM algorithm is an iterative algorithm that is utilized to solve problems which have interesting structure with respect to each individual optimization variable [17][Ch. 10]. The structure that is utilized to iteratively solve problem $\mathbb{P}_{0}$ is its pseudo-convexity and quasi-convexity with respect to each optimization variable $P_{s}(\gamma)$ and $T_{s}$.

\section{QUANTIZED SOFT-SENSING INFORMATION}

The impact of the SSI parameter, $\eta\left(\zeta, T_{s}\right)$, on the power adaptation, system energy consumption, and the interference toward PR has been shown earlier in Section III. However, in practical systems, it is difficult to continuously change the secondary transmission power. Furthermore, in the case of cooperative sensing systems, sharing a continuous change of the sensing information results in a large overload on the control channel [18]. Therefore, we propose a discretized (quantized) version of the SSI, such that the load of 


$$
\begin{aligned}
L= & \mathbb{E}\{t\} \\
& +\lambda_{0}\left[\mathbb{E}_{\gamma, \zeta \mid \mathcal{H}_{0}}\left\{T_{c} P_{t} \alpha_{0} P_{F A}^{c}\left(T_{s}\right) P_{s}(\gamma)\right\}+\mathbb{E}_{\gamma, \zeta \mid \mathcal{H}_{1}}\left\{T_{c} P_{t} \alpha P_{M D}\left(T_{s}\right) P_{s}(\gamma)\right\}+P_{c}+T_{s} P_{s n s}\right. \\
& \left.-\left[\mathbb{E}_{\gamma, \zeta \mid \mathcal{H}_{0}}\left\{t T_{c} \alpha_{0} P_{F A}^{c}\left(T_{s}\right) \log \left(1+P_{s}(\gamma) \gamma_{s}\right)\right\}+\mathbb{E}_{\gamma, \zeta \mid \mathcal{H}_{1}}\left\{t T_{c} \alpha P_{M D}\left(T_{s}\right) \log \left(1+\frac{P_{s}(\gamma) \gamma_{s}}{1+P_{p} \gamma_{p s}}\right)\right\}\right]\right] \\
& -\lambda_{1}\left[\mathbb{E}_{\gamma, \zeta \mid \mathcal{H}_{0}}\left\{T_{c} \alpha_{0} P_{F A}^{c}\left(T_{s}\right) \log \left(1+P_{s}(\gamma) \gamma_{s}\right)\right\}+\mathbb{E}_{\gamma, \zeta \mid \mathcal{H}_{1}}\left\{T_{c} \alpha P_{M D}\left(T_{s}\right) \log \left(1+\frac{P_{s}(\gamma) \gamma_{s}}{1+P_{p} \gamma_{p s}}\right)\right\}+R_{m i n}\right] \\
& +\lambda_{2}\left[\mathbb{E}_{\gamma, \zeta \mid \mathcal{H}_{1}}\left\{\alpha P_{M D}\left(T_{s}\right) P_{s}(\gamma) \gamma_{s p}\right\}-Q_{i n t}\right] .
\end{aligned}
$$

$$
\begin{aligned}
\lambda_{0} & {\left[\mathbb{E}_{\gamma, \zeta \mid \mathcal{H}_{0}}\left\{T_{c} P_{t} \alpha_{0} P_{F A}^{c}\left(T_{s}\right) P_{s}^{*}\right\}+\mathbb{E}_{\gamma, \zeta \mid \mathcal{H}_{1}}\left\{T_{c} P_{t} \alpha P_{M D}\left(T_{s}\right) P_{s}^{*}\right\}+P_{c}+T_{s} P_{s n s}\right.} \\
& \left.-\left[\mathbb{E}_{\gamma, \zeta \mid \mathcal{H}_{0}}\left\{t T_{c} \alpha_{0} P_{F A}^{c}\left(T_{s}\right) \log \left(1+P_{s}^{*} \gamma_{s}\right)\right\}+\mathbb{E}_{\gamma, \zeta \mid \mathcal{H}_{1}}\left\{t T_{c} \alpha P_{M D}\left(T_{s}\right) \log \left(1+\frac{P_{s}^{*} \gamma_{s}}{1+P_{p} \gamma_{p s}}\right)\right\}\right]\right]=0 . \\
\lambda_{1} & {\left[\mathbb{E}_{\gamma, \zeta \mid \mathcal{H}_{0}}\left\{T_{c} \alpha_{0} P_{F A}^{c}\left(T_{s}\right) \log \left(1+P_{s}^{*} \gamma_{s}\right)\right\}+\mathbb{E}_{\gamma, \zeta \mid \mathcal{H}_{1}}\left\{T_{c} \alpha P_{M D}\left(T_{s}\right) \log \left(1+\frac{P_{s}^{*} \gamma_{s}}{1+P_{p} \gamma_{p s}}\right)\right\}+R_{m i n}\right]=0 . }
\end{aligned}
$$

the continues SSI are discretly shared between the SUs, reducing the load on the channel.

As shown in (24), the impact of the sensing information is reflected by three parameters, $\eta\left(\zeta, T_{s}\right), P_{F A}^{c}\left(T_{s}\right)$, and $P_{M D}\left(T_{s}\right)$. Hereafter, we show the effect of quantizing the sensing information on these parameters. Consequently, their corresponding impact on the energy efficiency metric is shown. We begin by quantizing $\eta\left(\zeta, T_{s}\right)$ into $L$ discrete levels $\tilde{\eta}[l]$, where $\tilde{\eta}[l]$ is the quantized version, which is defined as,

$\Sigma_{\zeta}:\left\{\frac{l-1}{L} \eta_{\max }\left(\zeta, T_{s}\right)<\tilde{\eta}[l] \leq \frac{l}{L} \eta_{\max }\left(\zeta, T_{s}\right), \forall l \in 1, \ldots, L\right\}$,

where $\eta_{\max }\left(\zeta, T_{s}\right)$ denotes the maximum value of $\eta\left(\zeta, T_{s}\right)$, which occurs at $\zeta_{m}=\frac{\delta_{1}^{2} \mu_{0}-\delta_{0}^{2} \mu_{1}}{\delta_{1}^{2}-\delta_{0}^{2}}$, such that, $\eta_{\max }\left(\zeta, T_{s}\right)=\eta\left(\zeta_{m}, T_{s}\right)$. In this work, , without loss of generality, we consider L-ary uniform quantization level of $\eta\left(\zeta, T_{s}\right)$ [19]. It is shown that the corresponding quantized SSI $\tilde{\eta}[l]=\frac{2 l-1}{2 L} \eta_{\max }\left(\zeta, T_{s}\right), \quad \forall l \in\{1,2, \ldots, L\}$ if $\eta\left(\zeta, T_{s}\right) \in \Sigma_{\zeta}$. The associated quantized correctness and errorness probabilities are defined as $\tilde{P}_{F A}^{c}[l]=\operatorname{Pr}\left[\tilde{\zeta}_{\eta}[l] \leq \gamma_{t h} \mid \mathcal{H}_{0}\right]$ and $\tilde{P}_{M D}[l]=\operatorname{Pr}\left[\tilde{\zeta}_{\eta}[l] \leq \gamma_{t h} \mid \mathcal{H}_{1}\right]$. Note that $\tilde{\zeta}_{\eta}$ is the quantized $\zeta$ corresponds to $\tilde{\eta}$. Substituting $\tilde{\eta}[l]$ in (24), the quantized allocated power is expressed as,

$$
\tilde{P}_{s}^{*}[l]=\left[\frac{-\tilde{b}[l]+\sqrt{\left(\tilde{b}^{2}[l]-4 \tilde{a}[l] \tilde{c}[l]\right)}}{2 \tilde{a}[l]}\right]_{0}^{P_{p k}} .
$$

where, $\tilde{a}[l]=\gamma_{s}^{2} \tilde{\Phi}[l] ; \tilde{b}[l]=\tilde{\Phi}[l] \gamma_{s}\left(2+P_{p} \gamma_{p s}\right)-\gamma_{s}^{2} \alpha_{0} \tilde{P}_{F A}^{c}[l] \tilde{\eta}[l]-$ $\gamma_{s}^{2} \alpha \tilde{P}_{M D}[l] ; \tilde{c}[l]=-\gamma_{s} \alpha_{0} \tilde{P}_{F A}^{c}[l] \tilde{\eta}[l]\left(1+P_{p} \gamma_{p s}\right)-\gamma_{s} \alpha \tilde{P}_{M D}[l]+$ $\tilde{\Phi}[l]\left(1+P_{p} \gamma_{p s}\right)$. Then, $\tilde{\Phi}[l]=\frac{\tilde{\gamma}_{u}[l] \lambda_{0} P_{t} T_{c}+\lambda_{2} \bar{\gamma}_{s p} \alpha \tilde{P}_{M D}[l]}{T_{c}\left(\lambda_{1}+\lambda_{0} t\right)}$, and, $\tilde{\gamma}_{u}[l]=\left[\alpha_{0} \tilde{P}_{F A}^{c}[l] \tilde{\eta}[l]+\alpha \tilde{P}_{M D}[l]\right]$.

\section{APPLICATION AND Numerical RESUlts}

In this section, we begin by presenting the specific channels distributions of our analysis that is utilized in the numerical results. Then, we evaluate the proposed system versus several benchmarks using numerical results.

\section{A. Application on Specific Distributions}

The previous analysis is done for general fading channels. Hereafter, we use approximation method and assumed specific distributions of the channels and the sensing random variables. We assume that the fading channels $\gamma_{s}, \gamma_{s p}$, and $\gamma_{p s}$ are independent identically distributed random variables that follow an exponential distribution. Although $\zeta$ is not Gaussian, we use the central limit theorem to approximate it as Gaussian distribution which simplifies the analysis without loss of generality. This approximation is done for both hypotheses $\mathcal{H}_{0}$ and $\mathcal{H}_{1}$. Since we obtain a sufficiently large number of samples for $\zeta$, it is reasonable to use the Gaussian approximation in our analysis. The corresponding PDFs of both hypotheses are $g_{0}(\zeta)$ and $g_{1}(\zeta)$, and expressed as,

$$
g_{0}(\zeta)=\frac{\exp \left(-T_{s} \frac{\left(\zeta-\mu_{0}\right)^{2}}{2 \delta_{0}^{2}}\right)}{\sqrt{\left(2 \pi \delta_{0}^{2}\right)}}, g_{1}(\zeta)=\frac{\exp \left(-T_{s} \frac{\left(\zeta-\mu_{1}\right)^{2}}{2 \delta_{1}^{2}}\right)}{\sqrt{\left(2 \pi \delta_{1}^{2}\right)}},
$$

where, $\mu_{0}=1, \mu_{1}=P_{p} \bar{\gamma}_{p s}+1, \delta_{0}^{2}=2$, and $\delta_{1}^{2}=2\left(P_{p} \bar{\gamma}_{p s}+1\right)^{2}$.

\section{B. Simulation Results}

In this section we evaluate the proposed scheme versus the benchmark scheme and the quantized scheme via numerical results. We consider both evaluation metrics $E_{0}$ and $T_{s}$ versus several system parameters, namely, $\alpha, P_{F A}, Q_{i n t}$, and $L$. We consider the parameters mentioned in Table I to evaluate the systems. Note that the unit of the EPG metric is Joule per channel use per $T$.

TABLE I SIMULATION PARAMETERS.

\begin{tabular}{|l|l|}
\hline Parameter Name & Value \\
\hline Maximum PT power $\left(P_{p}\right)$ & $27 \mathrm{dBm}$ \\
\hline Maximum ST power $\left(P_{p k}\right)$ & $23 \mathrm{dBm}, 25 \mathrm{dBm}, 27 \mathrm{dBm}$ \\
\hline \# Monte-Carlo iterations & 10000 \\
\hline Wireless channels & $\begin{array}{l}\text { Rayleigh, } \\
\text { Slow Flat Fading }\end{array}$ \\
\hline$Q_{\text {int }}$ & $10 \mathrm{dBm}, 5 \mathrm{dBm}$ \\
\hline Minimum Rate $\left(R_{\text {min }}\right)$ & 1 \\
\hline$P_{t}$ & $1,0.5$ \\
\hline$P_{c}$ & 1 \\
\hline$P_{\text {sns }}$ & 1.5 \\
\hline
\end{tabular}

Figure 3(a) shows the effect of changing the false alarm probability on the normalized EPG metric. The evaluation is performed under two systems, proposed system (with SSI, noted as: "ss"), benchmark system (without SSI, noted as: "nss"). It is noted that increasing the false alarm probability reduces the normalized EPG metric. This result is expected, since the increment of $P_{F A}$ relaxes the problem. The increment of $P_{p}$ from $25 \mathrm{dBm}$ to $27 \mathrm{dBm}$ results in improving 


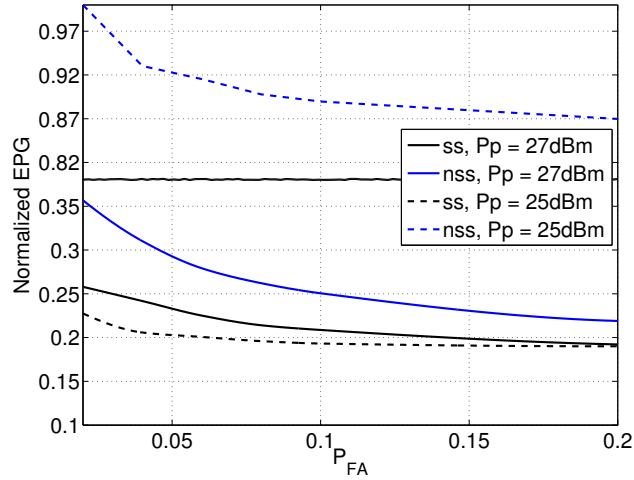

(a) The scheme with SSI, "ss", versus the scheme (b) Quantized EPG versus $L$ for $P_{F A}=0.1$ and $P_{F A}$ without SSI, "nss", for several values of $P_{p}$.

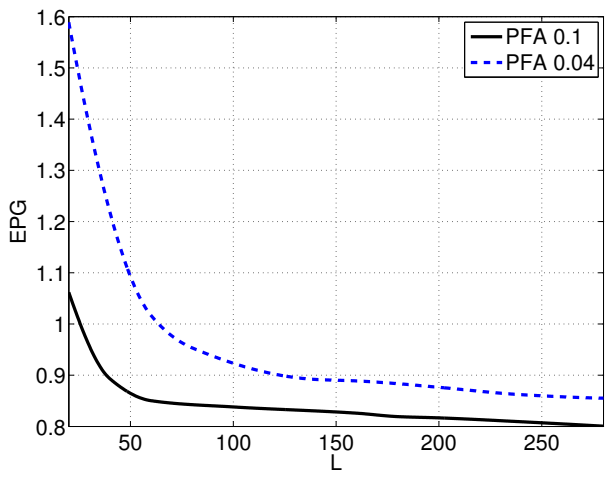

$=0.04$.

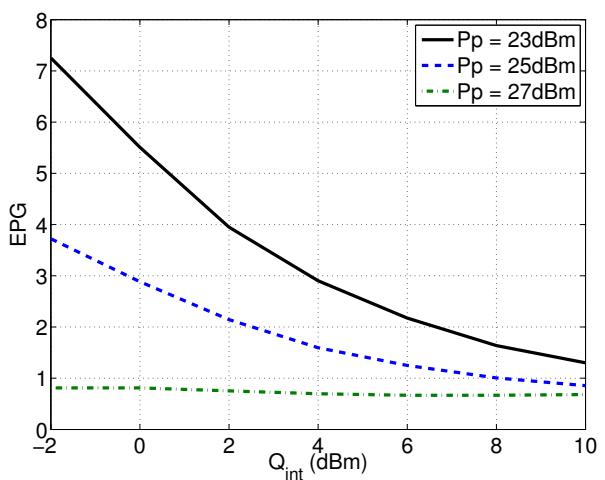

(c) EPG versus $Q_{\text {int }}$ for several $P_{p}$ values.

Fig. 3. All Numerical Results

the normalized EPG metric, about 0.67 units, under the without SSI system. This is an expected result since the only effect of sensing is through sensing accuracy which increases by increasing $P_{p}$. Whereas, increasing $P_{p}$ from $25 \mathrm{dBm}$ to $27 \mathrm{dBm}$ results in increasing the EPG metric, about 0.04 units, for the system with SSI. This can be interpreted by the fact that the SSI impact on the EPG is higher than the sensing accuracy effect, under the used parameters.

Figure 3(b) shows the effect of increasing the number of quantization levels on the performance of the EPG metric under both $P_{F A}$ $=0.1$ and $P_{F A}=0.04$. It is noted that increasing the quantization levels results in improving the EPG performance. The improvement range is about $0.9 \mathrm{EPG}$ units for $P_{F A}=0.04$, whereas its about 0.3 units for $P_{F A}=0.1$.

Finally, Figure 3(c) presents the EPG metric performance versus the change in the interference threshold $Q_{i n t}$. This evaluation is done for three values of primary transmission power $P_{p}=23$ $\mathrm{dBm}, 25 \mathrm{dBm}, 27 \mathrm{dBm}$. As expected, increasing $Q_{\text {int }}$ results in improving the EPG metric for all case. On the other hand, as stated earlier, increasing the $P_{p}$ values increases the sensing accuracy, which decreases the sensing time. Thus improving the EPG performance.

\section{CONCLUSION}

In this paper, we proposed a hybrid system with a mixture of energy efficiency and cognitive radio resource allocation. The proposed allocation scheme utilizes the soft-sensing information obtained from the secondary user sensor. The collected soft-sensing information is utilized to adapt the optimally allocated transmission power and the sensing duration of the secondary user. A quantization scheme of the soft-sensing information is provided to reduce the information sharing overload. The originally proposed problem is not clearly convex, therefore, we proved its pseudo-convexity structure to guarantee global optimal solution. Numerical and analytical evaluations of the proposed scheme are provided. Numerical results show that the softsensing information based system outperforms the one without softsensing information with up to 0.67 normalized energy per goodbit units. The increment of the quantization levels shows a system improvement up to 0.9 Joule per bits per channel use per $T$.

\section{REFERENCES}

[1] C. Han, T. Harrold, and et al., "Green radio: radio techniques to enable energy-efficient wireless networks," Communications Magazine, IEEE, vol. 49 , no. 6 , pp. $46-54$, june 2011.

[2] "Next generation/dynamic spectrum access/cognitive radio wireless networks: A survey," Computer Networks, vol. 50, no. 13, pp. 2127 - 2159, 2006.
[3] Z. Hasan, H. Boostanimehr, and V. Bhargava, "Green cellular networks: A survey, some research issues and challenges," Communications Surveys Tutorials, IEEE, vol. 13, no. 4, pp. 524 -540, quarter 2011.

[4] D. Feng, C. Jiang, G. Lim, J. Cimini, L.J., G. Feng, and G. Li, "A survey of energy-efficient wireless communications," IEEE Communications Surveys Tutorials, vol. 15, no. 1, pp. 167-178, 2013.

[5] G. Li, Z. Xu, C. Xiong, C. Yang, S. Zhang, Y. Chen, and S. Xu, "Energyefficient wireless communications: tutorial, survey, and open issues," IEEE Wireless Communications, vol. 18, no. 6, pp. 28-35, 2011.

[6] A. Alabbasi, Z. Rezki, and B. Shihada, "Energy efficiency and SINR maximization beamformers for spectrum sharing with sensing information," IEEE Transactions on Wireless Communications, 2014.

[7] R. S. Prabhu and B. Daneshrad, "An energy-efficient water-filling algorithm for OFDM systems," in IEEE International Conference on Communications (ICC), 2010, 2010, pp. 1-5.

[8] E. Peh, Y.-C. Liang, Y. L. Guan, and Y. Pei, "Energy-efficient cooperative spectrum sensing in cognitive radio networks," in Global Telecommunications Conference (GLOBECOM 2011), 2011 IEEE, Dec 2011, pp. 1-5.

[9] X. Li, J. Cao, Q. Ji, and Y. Hei, "Energy efficient techniques with sensing time optimization in cognitive radio networks," in IEEE Wireless Communications and Networking Conference (WCNC), 2013, April 2013, pp. 25-28.

[10] A. Alabbasi, Z. Rezki, and B. Shihada, "Energy efficient scheme for cognitive radios utilizing soft sensing," in IEEE Wireless Communications and Networking Conference (WCNC), 2014, pp. 1-6.

[11] Z. Shi, K. C. Teh, and K. H. Li, "Joint design of sensing and transmission in energy-efficient cognitive radio systems over fading channels," Communications, IET, vol. 7, no. 6, pp. 577-584, April 2013.

[12] A. Alabbasi, Z. Rezki, and B. Shihada, "Energy efficiency and SINR maximization beamformers for cognitive radio utilizing sensing information," in IEEE International Symposium on Information Theory (ISIT), 2014.

[13] F. F. Digham, M. Alouini, and M. K. Simon, "On the energy detection of unknown signals over fading channels," IEEE Transactions on Communications, vol. 55, no. 1, pp. 21-24, Jan. 2007.

[14] V. Asghari and S. Aissa, "Impact of detection uncertainties on the performance of a spectrum-sharing cognitive radio with soft sensing," IEEE Transactions on Vehicular Technology, vol. 61, no. 7, pp. 32723276, Sept 2012.

[15] A. Cambini and L. Martein, Generalized Convexity and Optimization: Theory and Applications. Springer, 2008.

[16] S. Boyd and L. Vandenberghe, Convex Optimization, 2004.

[17] R. W. Yeung, A First Course in Information Theory (Information Technology: Transmission, Processing and Storage). Secaucus, NJ, USA: Springer-Verlag New York, Inc., 2006.

[18] I. Akyildiz, W. Su, Y. Sankarasubramaniam, and E. Cayirci, "A survey on sensor networks," IEEE Communications Magazine, vol. 40, no. 8, pp. 102-114, Aug 2002.

[19] Y. Q. Shi and H. Sun, Image and Video Compression for Multimedia Engineering: Fundamentals, Algorithms, and Standards, 2nd ed. Boca Raton, FL, USA: CRC Press, Inc., 2008. 